\title{
Visual experimental study on development of mud film in slurry shield excavation face based on transparent soil technology
}

\author{
Henghui Liang ${ }^{1}$, Zhipeng $\mathrm{Shi}^{2}$, Xiaodong $\mathrm{Ni}^{2}{ }^{2}$, Songjie $\mathrm{Chen}^{3}$, and Qiankun Zeng ${ }^{4}$ \\ ${ }^{1}$ Wuxi Metro Group Co., Ltd, Wuxi 210096, Jiangsu, China \\ ${ }^{2}$ Hohai University, Nanjing 210000, Jiangsu, China
}

\begin{abstract}
In order to solve the problems of grouting diffusion and invisible formation process in shield slurry film forming test, transparent soil and transparent slurry were introduced to carry out shield mud film test based on shield grouting model. Using laser imaging combined with CCD camera (high-speed industrial camera) rapid capture photography technology, through a series of visible physical model tests of slurry diffusion and mud film development under different working conditions, the temporal and spatial conditions of slurry diffusion were observed and recorded in real time to explore the slurry diffusion law and the formation mechanism of mud film in slurry shield excavation face. The phenomenon shows that the smaller the grouting pressure is, the more favorable the formation of mud film is. The mud penetration distance is generally proportional to the grouting pressure.The infiltration distance of the grout increases with time, and the infiltration distance of the grout is similar at each moment under the same grouting pressure. The leachate volume and time show a quadratic function roughly under the same density and mud pressure, and the increase rate of the leachate volume begins to slow down with time.
\end{abstract}

Keywords: Mud film; Shield; Transparent soil; Visualization research.

\section{Introduction}

\subsection{Research Background}

Since 1843, when Thames Tunnel was first built under water by shield tunneling, predecessors have developed shield tunneling into a modern tunnel engineering technology with strong ground applicability, high degree of automation, environmental protection, economy, safety and efficiency through continuous summary and innovation of previous successful experiences.At present, shield technology has been widely used in the world's major engineering fields such as water conservancy, municipal administration, transportation and power.

\footnotetext{
*Corresponding author: lulingnxd@126.com
} 
Especially during the peak of infrastructure construction in China in the 21st century, the shield construction technology in our country got the unprecedented development opportunity. However, the stability of the soil and the variability of the section size greatly affect the construction of the shield method, and the current construction area mostly concentrated in urban areas such as bustling place, once appear in the process of the construction constraints instability phenomenon, will cause huge impact to the safety of the construction, the safety of personnel and equipment problems, and damage to property. How to ensure the stability of mud shield face is the focus of this paper.

\subsection{Research Status}

N. Horn ${ }^{[1]}$ obtained that the failure model of silo is wedge-shaped by the limit equilibrium method. Subsequently, G. Nagnostou et al. ${ }^{[2]}$ studied the stability of shield tunneling face by using the silo theory, and proposed the wedge failure mode of tunnel tunneling face. Later, E. Leca et al. ${ }^{[3]}$ proposed other different failure modes of tunnel excavation face. The above related shield excavation stability theories provide a certain theoretical support for the study of the formation mechanism of mud film on the shield excavation face. Vorobiev $^{[4]}$ considered the pressure redistribution in the process of mud film formation, established the corresponding mud film model, and predicted the process of mud film growth. Min et al. ${ }^{[5]}$ proved through experiments that the more mud particles content, the better the permeability of the mud film formed, and the particle content and particle size of mud are important factors affecting the mud quality. Takeshiwatanab and Hironobuyamazaki ${ }^{[6]}$ found that the addition of fine particle elements could reduce mud filtration volume, and studied the variation of mud filtration volume in the process of mud infiltration film formation through experiments. Heinz ${ }^{[7]}$ studied the relationship between the filtration loss of bentonite mud and formation stability in the high-permeability formation, and the results showed that the polymer content was closely related to the fluidity of bentonite mud, and the penetration distance of mud in the formation could be effectively reduced through the addition of filling materials such as sand and silt. Fritz ${ }^{[8]}$ carried out mud penetration film forming test in the formation with a permeability coefficient of $0.1 \mathrm{~cm} \cdot \mathrm{s}^{-1}$, and found that adding proper amount of particles and polymer additives in the mud was beneficial to the formation of mud film in the formation with high permeability. Han Xiaorui et al. ${ }^{[9]}$ used three kinds of mud with different viscosities to carry out permeability tests in the formation with a permeability coefficient of $0.04 \mathrm{~cm} \cdot \mathrm{s}^{-1}$, and concluded that the higher the mud viscosity, the better the physical stability of mud film under the condition of the same mud grading and density. Min, etc. ${ }^{[5]}$ made up of nine different mud, respectively, in five different coefficient of permeability of formation permeability test, mud osmosis film can be divided into three types (a mud, b mud cake + permeability zone, c permeability zone type), and the formation and calculation formula of average pore diameter is deduced based on judgment slurry infiltration type of method is proposed; Min etc. ${ }^{[10]}$ on a slurry shield with pressure tank formation stability problem, carried out the mud penetration test, first put forward using low density, low viscosity of the slurry to the excavation face penetration distance, formation permeability zone of mud membrane.

Comprehensive research, the status quo, more focused on the factors of mud membrane formation and mud membrane quality evaluation, etc., involving the mud membrane formation mechanism and the dynamic forming process of theoretical research and laboratory test is not perfect, so the grout diffusion regularity of mud and mud membrane membrane formation mechanism still need further research, provide theory support for the slurry shield tunneling construction. 


\section{Experimental design}

The purpose of the test is to observe the diffusion characteristics of the shield grouting test in real time with the help of transparent soil technology and optical observation technology and study the formation mechanism of mud film. Taking into account the invisible inside of ordinary natural soil materials and the visual characteristics of transparent soil materials, a complete set in order to device for visualization of penetration in the formation is developed. The following article will discuss the selection of transparent soil materials and the development of visualization devices.

\subsection{Selection of experimental variables}

\section{(1)Selection of particle size of transparent soil}

Transparent soil is the general name of transparent sand and transparent clay. At present, the synthetic transparent soil is mainly divided into two categories: the first one is composed of amorphous silica powder as aggregate and transparent clay, whose monomers can form large porous media with different particle sizes, whose properties are similar to those of natural clay. The second is composed of molten quartz sand as aggregate, which is similar to natural sand.After many trials, found that the fused quartz sand with high transparency and evenly distuibuted sizes and the nature of small linear expansion coefficient, so this experiment aggregate on the fused quartz sand as simulation transparent sand particle materials.Figure 1 shows molten quartz sand with different particle sizes:

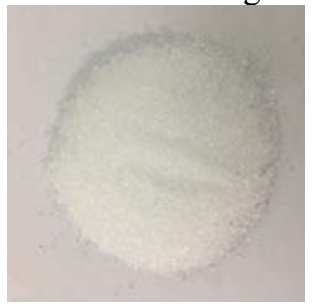

(a) $0.5-1 \mathrm{~mm}$

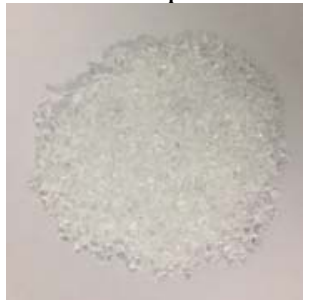

(b) $1-3 \mathrm{~mm}$

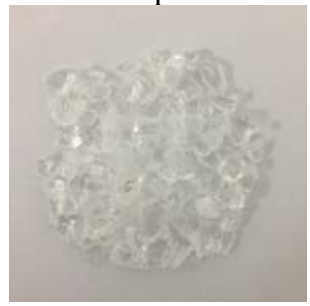

(c) $5-10 \mathrm{~mm}$

Fig. 1. Different sizes of quartz sand.

The transparency of model soil becomes worse as the particle size decreases. Too large a particle size makes the arrangement of soil particles relatively sparse, causing the slurry to quickly pass through the model soil and fail to form a mud film. In comprehensive consideration, the particle size range of $0.5-1 \mathrm{~mm}$ is selected in the experiment, which has good transparency and is suitable for forming a mud film.

(2) The choice of buried depth

The buried depth of the tunnel selected in the experiment is $C / D=0.5,1,2$, where $C$ is the buried depth of the tunnel, and D is the diameter of the shield. Visual observation of the formation process of the mud film on the excavation surface of the mud water shield with different depths is carried out.

(3) Selection of grouting pressure

Shield grouting needs to transmit the grout with a suitable grouting pressure. If the pressure is too small, it cannot guarantee the smooth infiltration of the grout into the transparent soil and fully fill the pores. If the pressure is too high, the grout will be instantly exposed to the excessive earth pressure in front of the soil and cause the grout. The upward movement caused the ground to bulge. Therefore, the experiment finally determines that the optimal pressure is $30-50 \mathrm{kPa}$.

(4) Selection of opening ratio 
Cutterhead opening ratio has an important influence on shield tunneling parameters. Different cutterhead opening ratios have different squeezing effects on the front of the excavation, which in turn affects the grouting form and grouting effect. The opening ratio is defined as the ratio of the opening area to the total area of the cutter head. The smaller the opening ratio, the greater the soil squeezing effect. This article refers to the selection principle of cutter head opening rate in actual engineering, and the selection range is $30 \%$ $50 \%$.

The experimental variables are shown in Table 1:

Table 1. Working condition type.

\begin{tabular}{|c|c|c|c|}
\hline Working condition & Burial depth ratio & Grouting pressure $(\mathrm{kPa})$ & Opening rate(\%) \\
\hline 1 & 0.5 & 30 & 40 \\
\hline 2 & 0.5 & 40 & 40 \\
\hline 3 & 0.5 & 50 & 40 \\
\hline 4 & 1 & 30 & 40 \\
\hline 5 & 1 & 40 & 30 \\
\hline 6 & 1 & 40 & 40 \\
\hline 7 & 1 & 40 & 50 \\
\hline 8 & 1 & 50 & 40 \\
\hline 9 & 2 & 30 & 40 \\
\hline 10 & 2 & 40 & 40 \\
\hline 11 & 2 & 50 & 40 \\
\hline
\end{tabular}

\subsection{Experimental equipment}

According to the similarity theory, the movement trend of soil particles is the focus of experimental research. If the shield machine and other components meet geometric similarity, synthetic transparent soil is similar to natural soil in some important physical properties, then transparent soil can be used as model soil. This test system satisfies the geometric similarity. It can be known that the transparent sand soil selected in this paper meets the similar requirements by carrying out related physical and mechanical tests on the transparent soil.

The model box is used as the stratum environment; the propulsion system simulates the mud water shield machine, the front of which is coaxially connected by two perforated discs, and the opening rate of the front cutter head during excavation of the shield machine is adjusted by rotation, and there is a mud chamber and a grouting pipe in between; The grouting system applies and controls the grouting pressure; the vacuum system is designed to mix the transparent soil aggregate with the pore liquid to remove the internal bubbles, because the laser refraction or reflection when passing through the bubbles in the transparent soil affects the transparent soil of the transparent soil material. This will further affect the visual observation of the experiment; use laser to image a certain surface of the soil in front of the shield, combine with CCD industrial camera to capture and record the image with high frequency, and then move the position of the laser through the displacement platform to obtain multiple cross-sectional images, and finally import the image into the computer Processing can obtain the movement trajectory of the particles in the transparent soil and synthesize the displacement cloud map, the filling form and diffusion range of the slurry at any time, and the film formation effect, and then analyze the formation mechanism of the mud film and the characteristics of the mud diffusion. The overall experimental device is shown in Figure 2. 


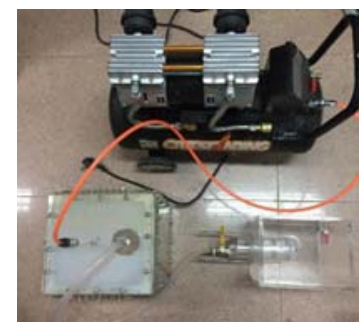

(a) Grouting device

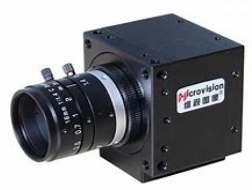

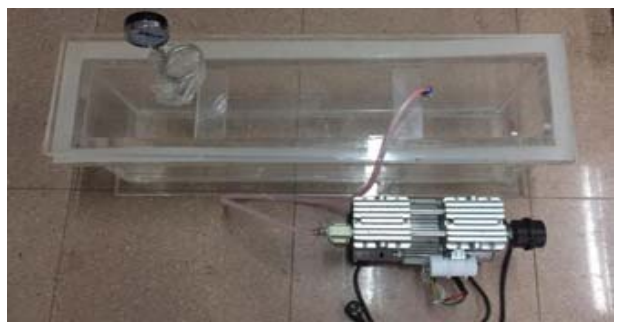

(b) Vacuum device

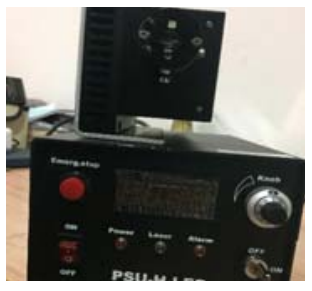

(c) Image acquisition system

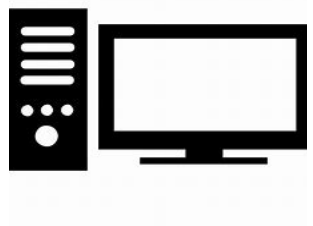

Fig. 2. Physical map of each test system.

\subsection{Experimental process}

(1) First assemble the test device according to the test requirements, check whether the air tightness of the grouting device is good, configure the slurry according to the appropriate solid-liquid ratio, add the tracer particles and stir evenly to prevent segregation. The configuration of $15 \#$ white oil and n-dodecane in the pore liquid was completed according to the mass ratio of $6.4: 1$.

(2) Transparent soil samples were filled.In order to prevent excessive air bubbles in the process of soil sample filling, the method of layered filling is adopted. Pore liquid was first added, and then solid particles were filled with a height of $2 \mathrm{~cm}$ each time to keep the liquid level higher than the solid surface. After each filling, glass rods were used to fully vibrate to eliminate bubbles, and then vacuum was carried out until the transparency was good. Repeat the above process until the height of the sample reaches the test requirements.

(3) The laser is turned on, the surface light source of the laser is adjusted to coincide with the longitudinal section of the shield center, and the CCD camera is set up at an appropriate height for imaging. Then the configured slurry is stirred evenly, and then poured into the slurry tank to quickly seal the slurry tank and prevent the slurry from segregation before grouting. Open the air compressor, open the grouting valve and start grouting after the pressure in the grout tank is constant. During the test, take pictures of longitudinal and cross-sectional grout diffusion at certain intervals, and analyze and study the diffusion process and final morphology of the grout in transparent soil.

\section{Experimental results and analysis}

\subsection{Experimental phenomena}

Taking slurry shield grouting in working condition 2 as an example, the seepage process of longitudinal and transverse grout is analyzed. Figure 3 shows the longitudinal section pictures taken at different moments in the slurry shield grouting process of working condition $2(\mathrm{C} / \mathrm{D}=0.5)$ and the slurry diffusion diagram of the actual project. 


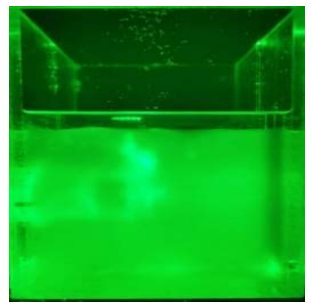

(a) $5 \mathrm{~min}$

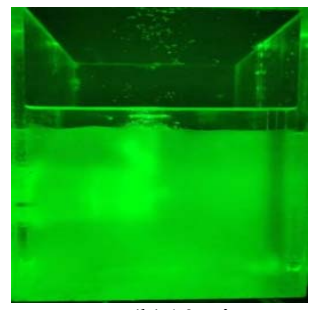

(b) $10 \mathrm{~min}$

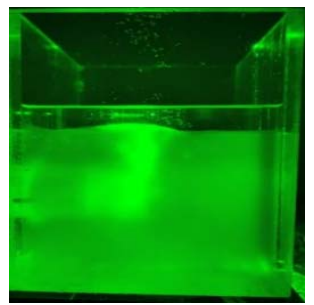

(c) $25 \mathrm{~min}$

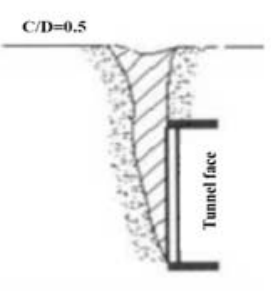

(d)project ${ }^{[11]}$

Fig. 3. Comparison of grout penetration form in longitudinal section and actual engineering.

At the beginning of grouting, the grout spreads towards the front of the shield under the action of the grouting pressure, and continuously fills the soil at a certain distance in front of the shield. When the filling reaches a certain level, the grout no longer spreads forward, but faces the cutter head. The outermost edge of the opposite surface spreads to the surroundings, which shows up and down spreading on the longitudinal section. The downward spreading area is formed by gravity. The existence of pore water pressure makes it difficult for the fine particles of the slurry to quickly penetrate into the pores under the action of gravity. Therefore, the downward diffusion area is very small; the earth pressure in the upper area is small, and the grout is easy to spread to the upper part of the shield. After spreading to a certain extent, the grout starts to spread along the spread area above the shield to the non-diffusion area, In the longitudinal section, it shows left and right diffusion, and the final diffusion form is similar to the actual project.

It is found in the experiment that the formation of the mud film is a dynamic development process. With the progress of the grouting process, the grout begins to penetrate into the soil. In the experiment, it is observed that the grout shows a trend of spreading from the center to the surroundings. Above the bottom edge of the shield machine.

\subsection{Analysis of experimental results}

In order to emphasize the different types of mud film that can be formed in the process of shield grouting, a comparative study was carried out on working conditions 1,8 and 10, because the types of mud film formed in these three working conditions are very different.Figure 4 shows the diffusion patterns of the slurry in the stratum with different burial depth ratios. It can be seen that the mud film formed when the burial depth ratio is 1 is the thickest, followed by that when the burial depth ratio is 2 , and that when the burial depth ratio is 0.5 is the thinnest.

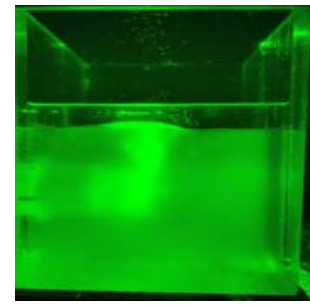

(a) 1

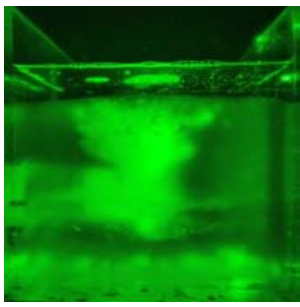

(b) 8

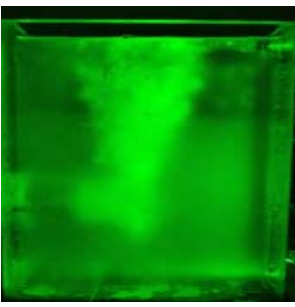

(c) 10

Fig. 4. Filling form of longitudinal section grout in the formation.

When the buried depth ratio is 0.5 and the grouting pressure is $30 \mathrm{kPa}$, the mud film formed can be regarded as the first type of mud film -- mud skin type mud film. When the buried depth ratio is 2 and the grouting pressure is $40 \mathrm{kPa}$, the mud film formed can be regarded as the second type of mud film -- mud skin + permeable zone type. When the 
buried depth ratio is 1 and the grouting pressure is $50 \mathrm{kPa}$, the mud film formed can be regarded as the third mud film type -- permeable zone type.

This paper carried out comparative tests under different buried depth ratios and different grouting pressures, and summarized the test results under different working conditions. After the test was completed, the slurry penetration distance was measured with a highprecision scale, and the slurry penetration distance at each time in the test process was shown in Figure 5.

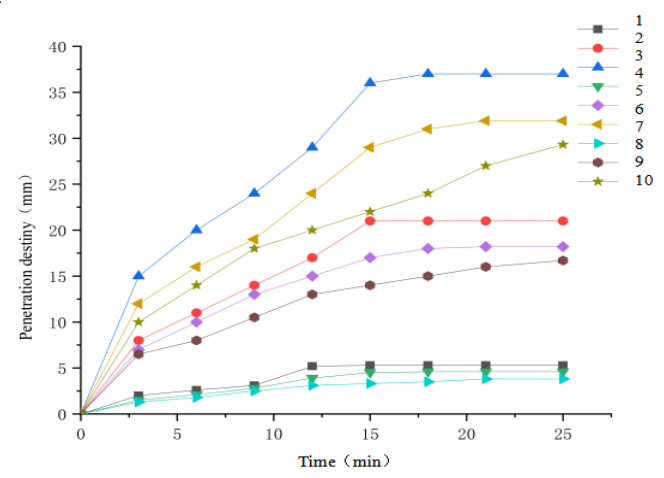

Fig. 5. Relationship between penetration distance and time under different working conditions.

As shown in Figure 6, the penetration distance of slurry increases with time. Under the same grouting pressure (working conditions 1, 4, 9; Operating conditions 2, 6, 10; In working conditions $3,8,11$ ), the penetration distance of grout is similar at all times, especially under low grouting pressure, and the ratio of buried depth has little effect on the penetration distance of grout.The penetration distance of slurry increases with the increase of grouting pressure, and the influence of grouting pressure on the penetration distance is significant.With the increase of grouting pressure, the difference of slurry penetration distance increases gradually under different buried depth ratio. Through working conditions 3,8 and 11, the increase of the buried depth ratio has a certain "delay effect" on the slurry diffusion, and this "delay effect" becomes more and more intense with the increase of the grouting pressure.

\section{Conclusion}

This article is based on technology and transparent soil slurry, the use of the development of visualization, slurry shield grouting experiment device of grouting and get the following conclusions:

(1)To develop a transparent slurry, a mass ratio of 5:15 \# 1 white oil with a mixture of ndodecane liquid, amorphous silica powder of 200 mesh to solid particles can be used to shield the visual model test grouting. In some working conditions, the cross section diffusion morphology was compared with the actual project, and it was found that the slurry diffusion morphology was similar, which confirmed the rationality of the slurry shield grouting test using the transparent slurry and the test method in this article.

(2)The mud penetration distance is generally proportional to the grouting pressure, and increasing the grouting pressure can significantly increase the mud penetration distance.The buried depth ratio of soil layer has little influence on the penetration distance of mud, while the grouting pressure has significant influence. The greater the grouting pressure(from $30 \mathrm{kpa}$ to $50 \mathrm{kpa}$ ), the greater the penetration distance.

(3)The penetration distance of the grout increases with time. Under the same grouting pressure, the penetration distance of the grout is similar at all times, especially under the 
low grouting pressure. Under the same grouting pressure, the ratio of burial depth has little effect on the penetration distance of the grout.The penetration distance of slurry increases with the increase of grouting pressure, and the influence of grouting pressure on the penetration distance is significant.With the increase of grouting pressure, the difference of slurry penetration distance increases gradually under different buried depth ratio. The increase of buried depth ratio has a certain "delay effect" on the diffusion of grout, and this "delay effect" becomes more and more intense with the increase of grouting pressure.

\section{References}

1. Janssen HA. Versuche uber Getre;ded ruck in Silozelle[J]. Zeitschriftdes Vereins deutscher Ingenieure, XXX-IX, 1895, (35): 1045-1049(in German).

2. Anagnostou G, Kovari K. The face stability of slury shield driven tunnels.tunneling and underground Space Technology, 1994, 9(2): 165-174.

3. Leca B, Bormieux L, Upper and lower bound solutions for the stability of shallow circular tunnel face[J]. ASCE ournal of Geotechnical Engineering, 1994(120): 11481165.

4. Zhang Gailing, Zhan Kaiyu, Gao Yue, et al. Comparative experimental investigation of chemical grouting into a fracture with flowing and static water. Mining Science and Technology, 2011, 21(2): 201-205.

5. Min Fanlu, Wei Daiwei, Jiang Teng, et al. Experimental study on the permeability characteristics of mud in stratum[J]. Rock and Soil Mechanics, 2014(10).

6. Cheng Zhanlin, $\mathrm{Wu}$ Zhongming, Xu Yanyong. Experimental study on stability of excavation surface of mud shield tunnel in sand foundation[J]. Journal of Yangtze River Scientific Research Institute,2001, 18(5):53-55

7. Heinz A Modifizierte Bentonitsuspensionen fur geotechbische Bauverfahren in Boden hoher Durchlassigkeit[D]. Swiss Federal Institute of Technology,2006

8. Fritz P. Additives for Slurry Shields in Highly Permeable Ground[J]. Rock Mechanic and Rock Engineering, 2007, 40(1): 81-95.

9. Han Xiaorui, Zhu Wei, Liu Quanwei, et al. The influence of mud properties on the quality of mud film formation on the excavation surface of a slurry shield [J]. Rock and soil mechanics, 2008, 29: 288-292.

10. Min Fanlu,Zhu Wei,Wei Daiwei,et al..Study on the change law of pore pressure of the excavation surface when the mud film is formed in the slurry shield[J]. Chinese Journal of Geotechnical Engineering, 2013, 35(004):722-727.

11. Liu Nian. Experimental study on stability of excavation face of shield tunneling in sandy soil[D]. Beijing traffic University, 2015 